This is the author's final, peer-reviewed manuscript as accepted for publication. The publisher-formatted version may be available through the publisher's web site or your institution's library.

\title{
Assessing the association between hoof thermography and hoof Doppler ultrasonography for the diagnosis of lameness in horses
}

Teresa L. Douthit, Jennifer M. Bormann, Nora M. Bello

\section{How to cite this manuscript}

If you make reference to this version of the manuscript, use the following information:

Douthit, T. L., Bormann, J. M., \& Bello, N. M. (2014). Assessing the association between hoof thermography and hoof Doppler ultrasonography for the diagnosis of lameness in horses. Retrieved from http://krex.ksu.edu

\section{Published Version Information}

Citation: Douthit, T. L., Bormann, J. M., \& Bello, N. M. (2014). Assessing the association between hoof thermography and hoof Doppler ultrasonography for the diagnosis of lameness in horses. Journal of Equine Veterinary Science, 34(2), 275-280.

Copyright: (c) 2014 Elsevier Inc.

Digital Object Identifier (DOI): doi:10.1016/j.jevs.2013.06.005

Publisher's Link: http://www.sciencedirect.com/science/article/pii/S0737080613004176

This item was retrieved from the K-State Research Exchange (K-REx), the institutional repository of Kansas State University. K-REx is available at http://krex.ksu.edu 


\section{Assessing the Association between Hoof Thermography and Hoof Doppler Ultrasonography for the Diagnosis of Lameness in Horses ${ }^{1}$}

Teresa L. Douthit PhD ${ }^{\mathrm{a}}$, Jennifer M. Bormann PhD, PAS ${ }^{\mathrm{a}}$, Nora M. Bello, PhD, VMD ${ }^{\mathrm{b}}$

${ }^{\mathrm{a}}$ Department of Animal Sciences \& Industry, Kansas State University, Manhattan, KS

${ }^{b}$ Department of Statistics, Kansas State University, Manhattan, KS

${ }^{1}$ This is contribution no. 13-254-J from the Kansas Agricultural Experiment Station, Manhattan.

Corresponding author: Teresa L. Douthit, PhD, Department of Animal Sciences \& Industry, Kansas State University, 129 Weber Hall, Manhattan, KS 66506. Telephone: (785) 532-1268. Fax: (785) 532-7059.

E-mail addresses: douthit@ksu.edu (T.L. Douthit), jbormann@ksu.edu (J.M. Bormann), nbello@ksu.edu (N.M. Bello). 


\begin{abstract}
The objectives of this study were to 1) assess the correlation between hoof surface temperature and ultrasonographic measurements of digital blood vessels in horses, and 2) evaluate their potential as predictors for clinical lameness. Twelve 3-year-old American Quarter Horses, 6 geldings and 6 mares, with average initial body weight of $459 \pm 31 \mathrm{~kg}$ were utilized. On days 0 , 30, 60, and 90 of the study, horses were weighed and subjected to clinical lameness exams. Doppler ultrasonography was used to measure diameter of the medial palmar artery in the distal left forelimb and velocity of blood flow through that artery starting at 60 min after morning feeding and repeated at 30-min intervals. Temperature measurements on the hoof were collected at 15-min intervals beginning 75 min after feeding using a digital thermographic camera. A series of bivariate linear mixed models were fitted to estimate the correlation between Doppler and temperature measurements. The within-horse and between-horse correlations between hoof surface temperature and velocity of blood flow in the distal limb through the medial palmar artery was estimated at $0.40(P>0.50)$ and $0.99(P<0.001)$, respectively. These results indicate that, at the horse level, the correlation between hoof temperature and velocity of blood flow in the distal limb was very high, but within horse, this correlation was not significantly different from zero. Velocity of blood flow at 60 min after feeding improved model fit to the lameness data, so was included as a model predictor for lameness.
\end{abstract}

Keywords: Doppler ultrasonography, Horse, Lameness, Thermography

\title{
1. Introduction
}

Lameness can be devastating to the utility of the horse, as the equine often is expected to perform athletically for many years. In fact, lameness has been cited not only as the most 
common cause of lost performance [1], but also the most frequently reported health problem in horses [2]. Recent studies have enhanced our understanding of the pathophysiology of lameness. In particular, altered digital circulation has been proposed to play a role, because circulating blood carries nutrients to the digital tissues [3,4], removes wastes, and is essential in the thermoregulation of the hoof [4]. In addition, the rigid nature of the hoof wall makes the equine foot particularly susceptible to changes in pressure, with little room for expansion to accommodate the edema that characterizes any inflammatory process [4]. Both navicular syndrome and laminitis, common equine lamenesses, have been associated with altered circulation in the equine hoof [3-8].

Doppler ultrasonography and thermographic imaging are noninvasive methods of assessing blood flow to various tissues within the body. Moving structures, such as erythrocytes in circulating blood, show differential patterns of sound wave reflectance based on their speed of travel; these patterns can be detected through Doppler ultrasonography [9]. Doppler ultrasonography has been utilized to evaluate blood flow throughout the equine limb $[10,11]$ and the equine hoof specifically [12]. Studies have shown that despite considerable variability in measurements, the use of Doppler ultrasonography in the equine limb is sufficiently repeatable and sensitive to detect changes in blood flow $[13,14]$. In fact, Doppler ultrasonography is able to detect differences as small as 0.005 and $0.01 \mathrm{ml} / \mathrm{min}$ in digital arterial and venous flow [13], making the technique sufficiently sensitive to detect different physiological stages [15].

Thermographic imaging is commonly used to measure surface temperature, which in turn may be considered an indicator of blood flow to an area. As local circulatory flow in the equine limb increases, so does the emission of infrared energy [16]. This technique has been shown to yield repeatable measurements [17], although ambient temperature differences may affect 
measurements obtained [18]. Thermography has been used to detect a variety of inflammatory conditions [19] and early signs of osteoarthritis [20] in the horse. The use of thermography requires little operator training and does not require close proximity to a blood vessel, making it more practical than Doppler ultrasonography to assess blood flow in areas such as the hoof. Despite the fact that thermographic imaging is relatively simple and straightforward to administer, it is not used widely in clinical settings [21].

The objectives of this study were to 1) assess the correlation between hoof surface temperature and ultrasonographic measurements of digital blood vessels in horses, and 2) evaluate their potential as predictors for clinical lameness.

\section{Materials and Methods}

All procedures were approved by the Institutional Animal Care and Use Committee at Kansas State University. This study was conducted concurrently and with the same set of horses assigned to another experiment designed to evaluate the effects of endophyte-infected fescue consumption on digital circulation in the horse [11]. In brief, 12 3-year-old American Quarter Horses, 6 geldings and 6 mares, with mean initial body weight of $459 \pm 31 \mathrm{~kg}$ were blocked by weight, gender, and Hyperkalemic Periodic Paralysis (HYPP) status (homozygous normal or heterozygous) and assigned to dietary treatment groups as described in Douthit et al. [11]. The effect of dietary treatment on digital circulation was reported in Douthit et al. [11]. This manuscript addresses the relationships between the measurements of digital circulation to each other, as well as their relationship to lameness, independent of treatment, which were not addressed in the initial publication [11]. 
Horses were housed in 45-m x 20-m drylot pens and received routine vaccinations, deworming, and hoof care. The study was conducted June through October 2007. Horses were weighed and subjected to clinical lameness examinations by a board-certified equine veterinarian on days -1 and 0,30 and 31, 60 and 61, and 90 and 91 of the study. One veterinarian conducted all examinations on days -1 through 31, with another veterinarian conducting all remaining examinations. On each of these days, lameness was assessed by the veterinarian after observing horses trotting on a straight line, horses trotting a circle on a longe line on a concrete surface, and their sensitivity to hoof testers. Initially horses were scored according to the Obel grading system, and then any horse receiving a grade greater than 0 (those showing any sign of lameness) were scored 1 , and sound horses were scored 0 . Concurrently with the clinical exams, surface temperature of each horse’s front hooves was recorded throughout the day using a digital thermographic camera, ThermaCAM P45HSV (FLIR Systems, Inc., Pleasant Hill, MO). The temperature of each front hoof was measured by a trained graduate student, and readings were averaged between hooves for each horse at each time point within day. Temperature measurements were collected approximately $1 \mathrm{~cm}$ below the coronary band at 15-min intervals beginning 75 min after feeding. Doppler ultrasonography (Megas ES FD570A; Biosound Esaote, Inc.) was used to measure diameter of the medial palmar artery in the distal left forelimb and velocity of blood flow through that artery. Each series of measurements took place on two consecutive days, with six horses evaluated each day. Approximately $12 \mathrm{~h}$ prior to measurement, horses were placed in climate-controlled stalls. Sampling with Doppler ultrasonography began 60 min after morning feeding and was repeated at 30-min intervals by the same veterinarian that conducted the lameness examinations. Velocity was recorded on all collection dates, but diameter was not collected on day (d) 0 . Volume of blood flow was calculated by multiplying 
velocity by diameter. Further details about management of the horses and measurement of responses can be found in Douthit et al. [11].

\subsection{Statistical Analysis}

For the purpose of exploring the association between surface temperature and ultrasonographic measurements on the hoof, a series of three bivariate linear mixed models were fitted to hoof temperature and velocity of blood flow, hoof temperature and diameter of medial palmar artery, and hoof temperature and volume of blood flow. For each of the responses of interest, namely velocity, diameter, volume, and temperature, the arithmetic mean of all measurements taken on a horse on a given day was calculated, resulting in a single data point for each response variable per horse and day. The statistical model included fixed effects of treatment, day, and their two-way interaction, as well as a random effect of horse nested within treatment to account for repeated measures over multiple days for each horse. All variance components were estimate using residual maximum likelihood. Statistical models were fitted using the MIXED procedure of SAS (Version 9.2, SAS Institute, Cary, NC). The residual (i.e., within-horse) and random (i.e., between-horse) variance-covariance matrices were specified as unstructured to allow for separate estimation of variance and covariance parameters at both levels. These variance-covariance estimates were used to estimate the within-horse and betweenhorse correlation coefficients between hoof temperature and velocity of blood flow, hoof temperature and diameter of medial palmar artery, and hoof temperature and volume of blood flow. These correlation coefficients were then tested against a null value zero using a t-test, as described in Kutner et al. [22]. Because diameter and volume were not measured on d 0, there were only 3 repeated measures for these variables; therefore the correlations between hoof 
temperature and diameter of medial palmar artery, and hoof temperature and volume of blood flow could not be estimated.

The association between occurrence of lameness (yes/no) and velocity, diameter, volume, and temperature measurements on the hoof was also examined. A generalized linear mixed model was used to describe the association between lameness and the predictors of interest; the model was determined by step-wise forward selection using maximum-likelihood-based Bayesian Information Criterion (BIC) as an indicator of model fit. The base model included day, treatment, and sex as fixed effects and the random effect of horse nested within treatment and sex. Candidate explanatory variables evaluated for model inclusion were velocity, diameter, and volume measured at times $60,90,120,150$, and 180 min, and temperature measured at times 75 , 90, 105, 120, 135, 150, and 165 min after feeding. For this analysis, temperature, velocity, diameter, and volume were not averaged within horse and day. Model fitting was conducted using the GLIMMIX procedure of SAS (Version 9.2, SAS Institute, Cary, NC), with $P=0.05$ as the significance level. No horses showed lameness on d 0 ; therefore, only observations from $\mathrm{d}$ 30, 60, and 90 were used in this analysis to prevent quasi-complete separation of data points.

\section{Results}

\subsection{Relationship between Doppler ultrasound and temperature measurements}

Scatterplots of observed values for hoof surface temperature and velocity of blood flow are presented in Figures 1 and 2, respectively, for each horse and are color-coded for d 0, 30, 60, and 90. The estimated within-horse (i.e., residual level) correlation between hoof surface temperature and velocity of blood flow in the distal limb through the medial palmar artery was $0.40(P>$ 0.50); that is, for a given horse and within a given day, measurements of hoof temperature and 
velocity of blood flow in the distal limb were only moderately correlated. The within-horse relationship between hoof surface temperature and velocity of blood flow is depicted in Figure 3 by means of a scatterplot of their respective residuals.

The estimated between-horse (i.e., random level) correlation was $0.99(P<0.001)$; that is, after estimating a relative horse-specific response over the course of the study (much as is done with best linear unbiased prediction in linear mixed models), the estimated horse-level correlation for hoof surface temperature and velocity of blood flow in the distal limb was of considerable magnitude. Figure 4 illustrates this relationship using a scatterplot of the estimated horse-specific differential effects on hoof temperature over estimated horse-specific differential effects on blood flow velocity.

In the remaining two bivariate linear mixed models fitted to hoof surface temperature and each of diameter and volume, per hoof blood vessel ultrasonographic measurements, betweenhorse variance components estimation converged to 0 ; therefore, it was not possible to compute the between-horse correlation between variables.

\subsection{Relationship of hoof temperature and Doppler ultrasound measurements with} lameness

When BIC-based forward model selection was used to assess explanatory variables associated with lameness, none of the variables were significantly $(P<0.05)$ associated with lameness. Velocity of blood flow at $60 \mathrm{~min}$ after feeding was identified as the best-fitting predictor of lameness (decreased the BIC by more than 2) after accounting for other variables in the model, namely day, treatment, and sex. In addition, hoof temperature at 75, 90, 105, 120, 
135, 150 min after feeding or average hoof temperature seemed to have comparable model fit to the data relative to velocity at 60 min, meaning they decreased the BIC by approximately the same amount and had similar $P$-values. However, after one of these explanatory variables was added, none of the others added to the predictive ability of the model. Other studies have reported success in diagnosing lameness in horses with the aid of thermography $[25,26]$. In our case, even though model selection indicated that several explanatory variables (as listed above) contributed to explaining lameness, their $P$-values ranged from 0.13 to 0.18 . The estimated $95 \%$ confidence intervals on the odds ratios for velocity, volume, diameter, or temperature when incorporated into the base model included the null value 0 in all cases and had $P$-values $>0.13$, indicating that none of the variables were significantly associated with the odds of lameness.

\section{Discussion}

The moderate correlation detected between surface temperature of the hoof and velocity of blood flow concurs with the conclusion drawn by other authors that Doppler and thermographic readings show a poor degree of correlation $[23,24]$. Such moderate correlation might be attributable to measurement noise associated with the equipment or the operator as well as variability due to environmental or time-of-day effects; however, an effort was made to minimize environmental interference by keeping the horses stalled in the air-conditioned environment for at least $12 \mathrm{~h}$ prior to initiating thermographic measurements. Also, all measurements were taken indoors at a constant temperature and without the interference from direct sunlight or wind. 
Because the estimated horse-level correlation for hoof surface temperature and velocity of blood flow in the distal limb was of considerable magnitude, it appears that at the horse level whereby multiple measurements on a given horse are considered jointly, hoof surface temperature may be expected to serve as a reliable proxy for Doppler-measured velocity of blood flow for a horse and vice versa. Similar to our findings, Wongaumnauykul et al. [27] reported no evidence of association between Doppler parameters and degree of lameness. The limited power from the small size of the dataset may have prevented us from identifying predictors with small effect sizes.

\section{Conclusions}

The very high between-horse correlation between velocity of blood flow through the medial palmar artery and hoof temperature indicate that hoof temperature can be used to predict velocity of blood flow on a horse level basis; however, considerable measurement noise is associated with repeating observations within a given animal. No significant associations between velocity, diameter, volume, or temperature and lameness were found. However, including velocity of blood flow through the medial palmar artery at 60 min after feeding; hoof temperature at $75,90,105,120,135$, and 150 min after feeding; or average hoof temperature into the model to predict lameness seemed to enhance model fit to the data despite non-significant results. Further research with a larger sample size is needed to replicate these results and explore other associations with suitable statistical power. 


\section{References}

[1] Jeffcott LB, PD Rossdale, J Freestone, CJ Frank, PF Towers-Clark. An assessment of wastage in thoroughbred racing from conception to 4 years of age. Equine Vet J 1982;14:185-98. [2] Kaneene JB, WA Ross, R Miller. The Michigan equine monitoring system. II. Frequencies and impact of selected health problems. Prev Vet Med 1997;29:277-92.

[3] Harris P, SR Bailey, J Elliott, A. Longland. Countermeasures for pasture-associated laminitis in ponies and horses. J Nutr 2006;136:2114S-21S.

[4] Robinson NE. Step by painful step: Increasing knowledge about laminitis. Equine Vet J 1998;30:89-90.

[5] Hood DM, DA Grosenbaugh, MB Mostafa, SJ Morgan, BC Thomas. The role of vascular mechanisms in the development of acute equine laminitis. J Vet Intern Med 1993;7:228-34. [6] Peroni JF, JN Moore, E Noschka, ME Grafton, M Aceves-Avila, SJ Lewis, et al. Predisposition for venoconstriction in the equine laminar dermis: Implications in equine laminitis. J Appl Physiol 2006;100:759-63.

[7] Pollitt CC, CT Davies. Equine laminitis: Its development coincides with increased sublamellar blood flow. Equine Vet J 1998;26:125-32.

[8] Rijkenhuizen AB. Navicular disease: A review of what's new. Equine Vet J 2006;38:82-8. [9] Weyman AE. Principles of Doppler flow measurement. Principles and practice of echocardiography. 2nd ed. Pennsylvania: Lea and Febiger 1994, p. 143-62.

[10] Cochard T, RL Toal, AM Saxton. Doppler ultrasonographic features of thoracic limb arteries in clinically normal horses. Am J Vet Res 2000;61:183-90. 
[11] Douthit TL, JM Bormann, KC Gradert, LW Lomas, SF DeWitt, JM Kouba. The impact of endophyte-infected fescue consumption on digital circulation and lameness in the distal thoracic limb of the horse. J Anim Sci 2012;90:3101-11.

[12] Hoffmann KL, AKW Wood, PH McCarthy, KA Griffiths, DL Evans, RW Gill. 1999.

Sonographic observations of the peripheral vasculature of the equine thoracic limb. Anat Histol Embryol 1999;28:281-9.

[13] Menzies-Gow NJ, CM Marr. Repeatability of Doppler ultrasonographic measurement of equine digital blood flow. Vet Radiol Ultrasound 2007;48(3):281-5.

[14] Raisis AL, LE Young, HB Meire, PM Taylor, K Walsh, P Lekeux. Variability of Doppler ultrasound measurements of hindlimb blood flow in conscious horses. Equine Vet $\mathrm{J}$ 2000;32:125-32.

[15] Menzies-Gow NJ, MF Sepulveda, SR Bailey, FM Cunningham, J Elliott. Roles of thromboxane a(2) and 5-hydroxytryptamine in endotoxin-induced digital vasoconstriction in horses. Am J Vet Res 2008;69:199-207.

[16] Stromberg B, I Norberg. Infra-red emission and ${ }^{133}$ Xe-disappearance rate studies in the horse. Equine Vet J 1971;1:1-7.

[17] Palmer SE. Use of the portable infrared thermometer as a means of measuring limb surfacetemperature in the horse. Am J Vet Res 1981;42:105-8.

[18] Mogg KC, CC Pollitt. Hoof and distal limb surface-temperature in the normal pony under constant and changing ambient-temperatures. Equine Vet J 1992;24:134-9.

[19] Purohit RC, MD Mccoy. Thermography in the diagnosis of inflammatory processes in the horse. Am J Vet Res 1980;41:1167-74. 
[20] Vaden MF, RC Purohit, MD Mccoy, JT Vaughan. Thermography - a technique for subclinical diagnosis of osteo-arthritis. Am J Vet Res 1980;41:1175-9.

[21] Rantanen NW. Equine imaging modalities: An algorithm approach. AAEP Proceedings 2010;56:290-6.

[22] Kutner, MH, CJ Nachtsheim, J Neter, W Li. Applied Linear Statistical Models. $5^{\text {th }}$ ed. London: McGraw-Hill Irwin; 2004, p. 84.

[23] Seifalian AM, G Stansby, A Jackson, K Howell, G Hamilton. Comparison of laser Doppler perfusion imaging, laser Doppler flowmetry, and thermographic imaging for assessment of blood flow in human skin. Eur J Vasc Surg 1994;8:65-9.

[24] Wienzek H, H Freise, I Giesler, HK Van Aken, AW Sielenkaemper. Altered blood flow in terminal vessels after local application of ropivacaine and prilocaine. Reg Anesth Pain Med 2007;32:233-9.

[25] Eddy A, V Hoogmoed, R Synder. The role of thermography in the management of equine lameness. Vet J 2001;162:172-81.

[26] Turner TA. Diagnostic thermography. Vet Clin North Am Equine Pract 2001;17:95-113.

[27] Wongaumnuaykul S, C Siedler, H Schobesberger, C Stanek. Doppler sonographic evaluation of the digital blood flow in horses with laminitis or septic pododermatitis. Vet Radiol Ultrasound 2006;47:199-205. 
Figure 1. Scatterplot of observed hoof temperature $\left({ }^{\circ} \mathrm{F}\right)$ on days -1 and 0,30 and 31,60 and 61 , and 90 and 91 for 12 horses. Depicted are daily observations (*) and means ( $\bullet$ ) for each horse.

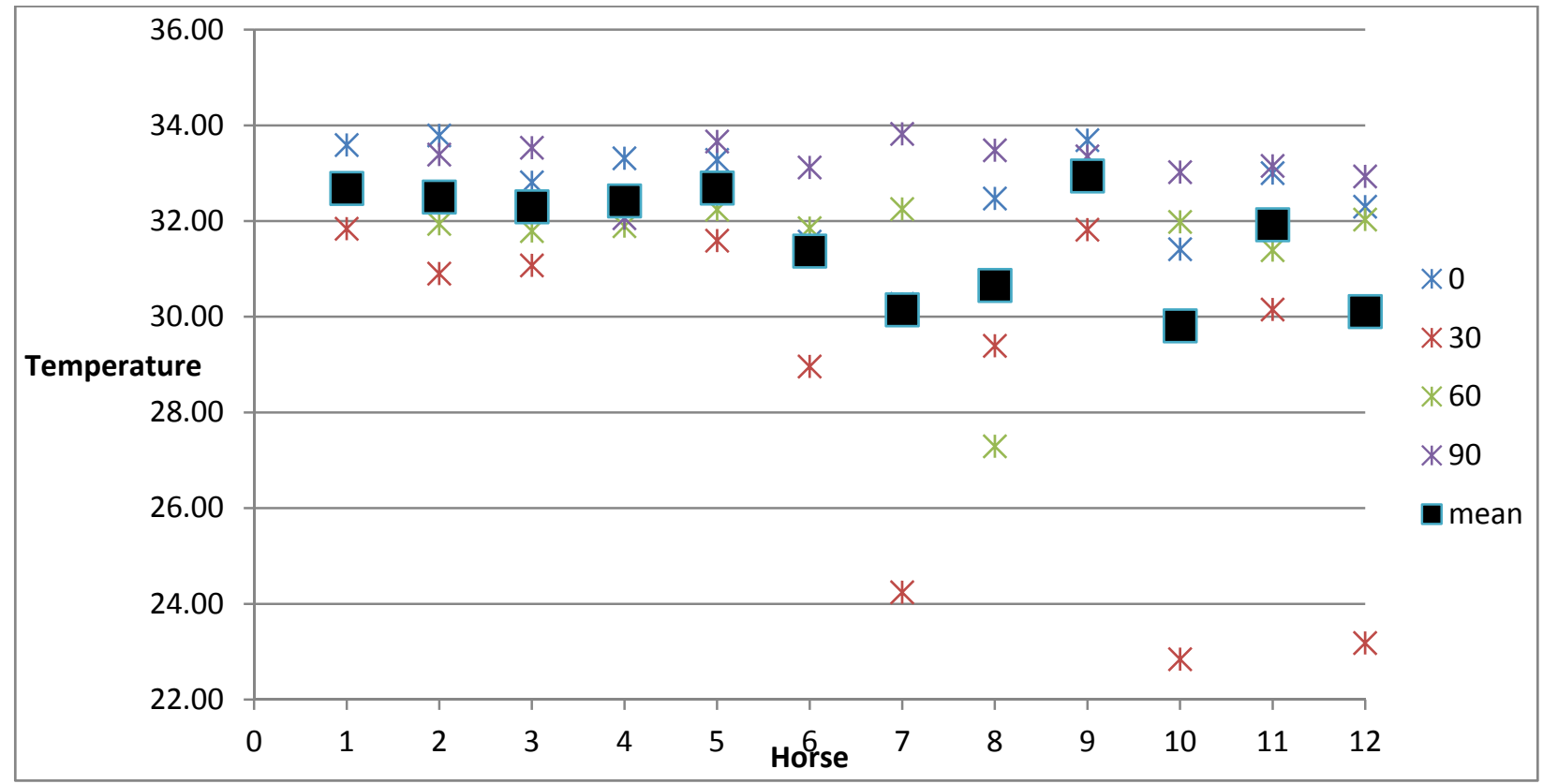


Figure 2. Scatterplot of observed velocity of blood flow $(\mathrm{m} / \mathrm{s})$ as measured by Doppler ultrasonography through the medial palmar artery on days - 1 and 0,30 and 31, 60 and 61, and 90 and 91 for 12 horses. Depicted are daily observations (*) and means ( $\boldsymbol{\square})$ for each horse.

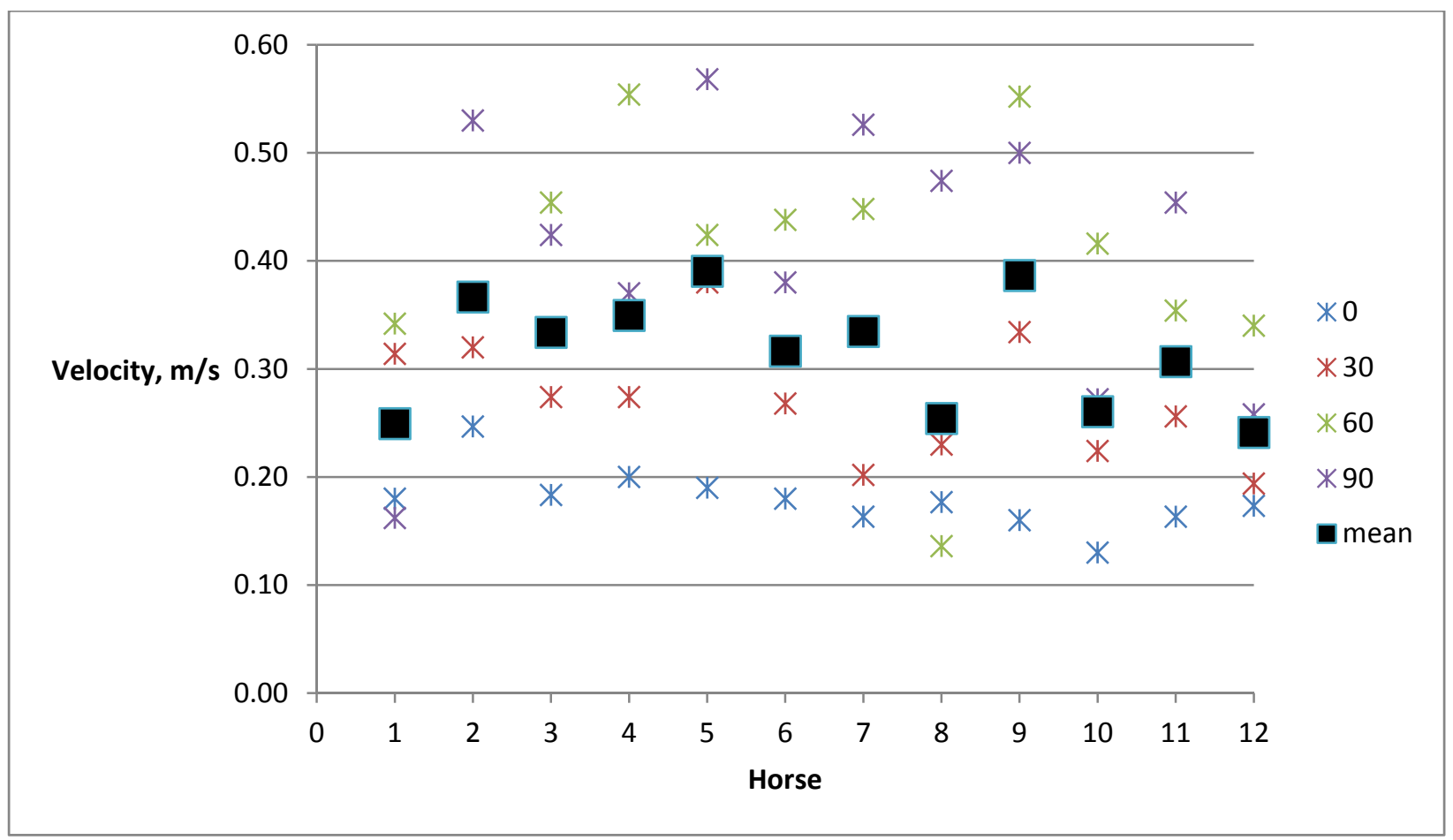


Figure 3. Scatterplot of within-horse residuals (color-coded within horse) velocity of blood flow through the medial palmar artery (m/s; y-axis) as measured by Doppler ultrasonography and hoof surface temperature $\left({ }^{\circ} \mathrm{C}\right.$; $\mathrm{x}$-axis). The estimated within-horse correlation was $0.40(P<0.02)$.

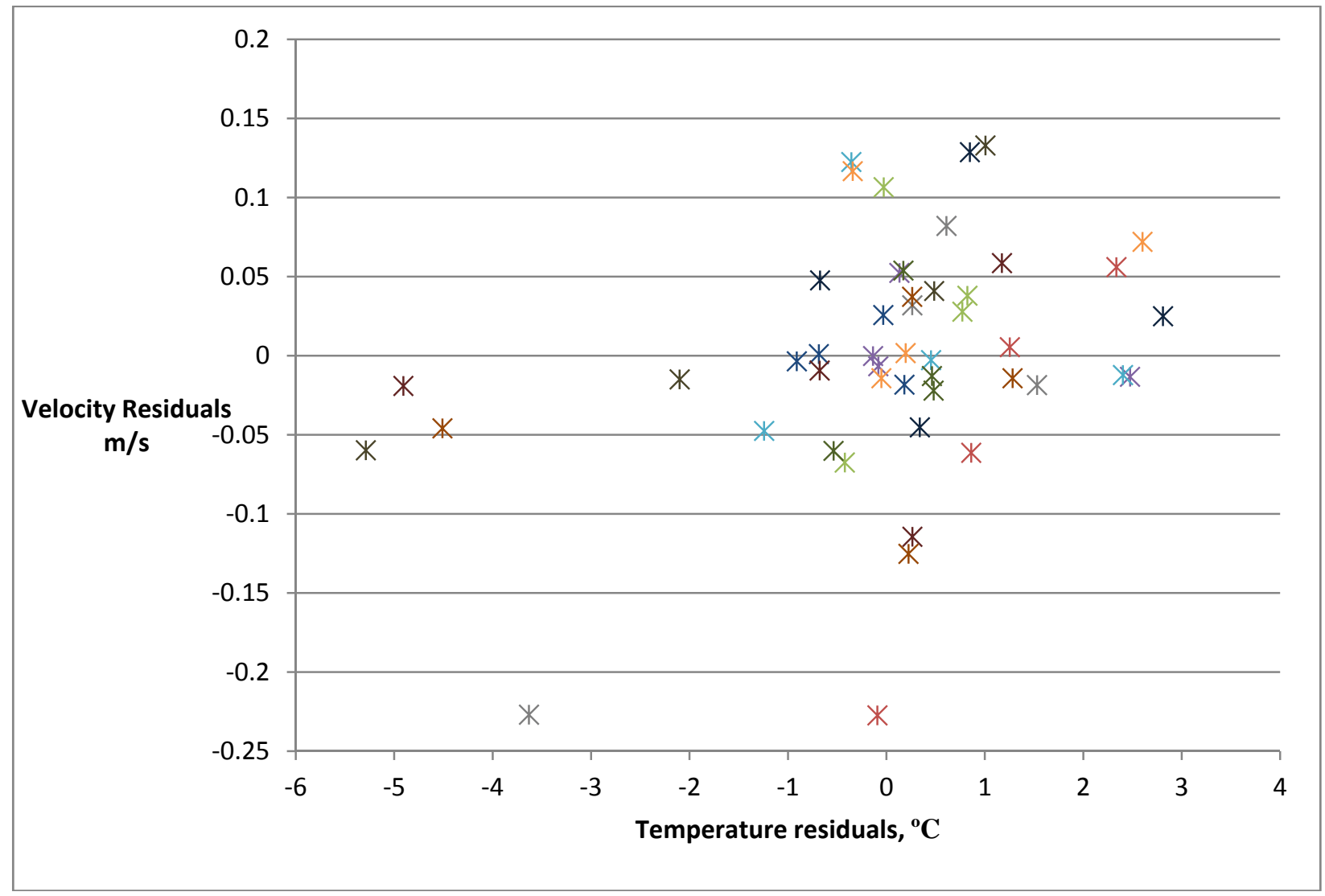


Figure 4. Scatterplot of horse-level (i.e., random) effects for velocity of blood flow in the medial palmar artery $(\mathrm{m} / \mathrm{s})$ as measured by Doppler ultrasound and hoof surface temperature $\left({ }^{\circ} \mathrm{F}\right)$, relative to an overall horse effect of zero.

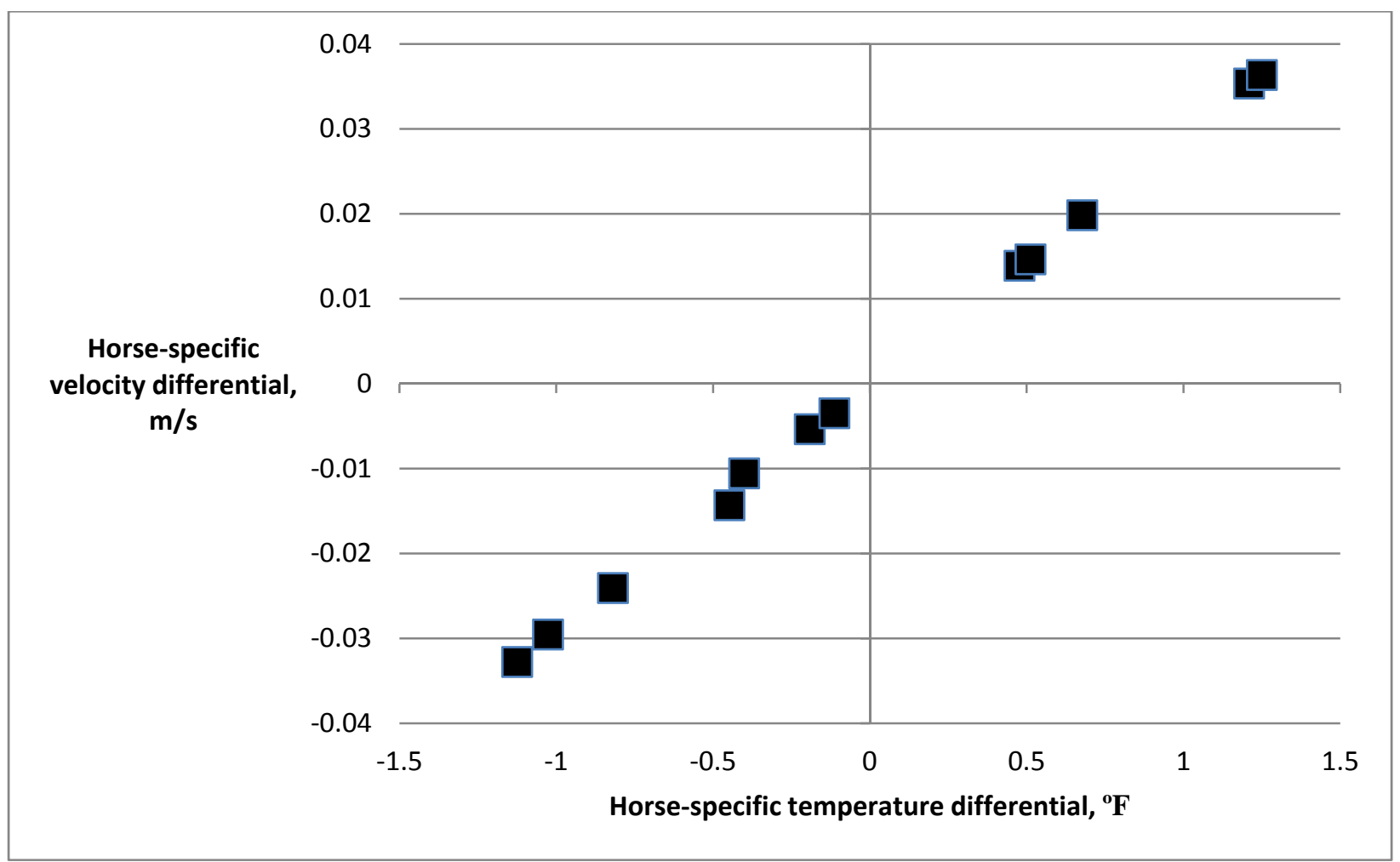

